\title{
Stage IVB Cervical Cancer AJCC v8
}

National Cancer Institute

\section{Source}

National Cancer Institute. Stage IVB Cervical Cancer AJCC v8. NCI Thesaurus. Code C139755.

Stage IVB includes: Any T, Any N, M1. M1: Distant metastasis (including peritoneal spread or involvement of the supraclavicular, mediastinal, or distant lymph nodes; lung; liver; or bone). (from AJCC 8th Ed.) 